\title{
Design of Multilingual Speech Synthesis System
}

\author{
S. SARASWATHI ${ }^{1}$, R. VISHALAKSHY ${ }^{2}$ \\ ${ }^{1}$ Assistant Professor, Department of Information Technology, Pondicherry Engg., College, Pondicherry, India \\ ${ }^{2}$ Department of Information Technology, Pondicherry Engg., College, Pondicherry, India \\ Email:swathimuk@yahoo.com
}

\begin{abstract}
The main objective of this paper is to convert the written multilingual text into machine generated synthetic speech. This paper is proposed in order to provide a complete multilingual speech synthesizer for three languages Indian English, Tamil and Telugu. The main application of TTS system is that it will be helpful for blind and mute people that they could have the text read to them by computer. TTS system will help in retrieving the information from sites that contain information in different languages. It can be used in educational institutions for pronunciation teaching of different languages. We use concatenative speech synthesis where the segments of recorded speech are concatenated to produce the desired output. We apply prosody which makes the synthesized speech sound more like human speech. Smoothing is also done to smooth the transition between segments in order to produce continuous output. The Optimal Coupling algorithm is enhanced to improve the performance of speech synthesis system.
\end{abstract}

Keywords: Prosody, Smoothing, Optimal Coupling

\section{Introduction}

Speech synthesis is the artificial production of human speech. A computer system used for this purpose is called a speech synthesizer, and can be implemented in software or hardware. A text-to-speech (TTS) system converts normal language text into speech; other systems render symbolic linguistic representations like phonetic transcriptions into speech. Synthesized speech can be created by concatenating pieces of recorded speech that are stored in a database. Systems differ in the size of the stored speech units. The speech units can be phonemes, diphones, syllables etc. For specific usage domains, the storage of entire words or sentences allows for high-quality output.

A text-to-speech system is composed of two parts: a front-end and a backend. The front-end has two major tasks. First, it converts raw text containing symbols like numbers and abbreviations into the equivalent words. This process is often called text normalization, Preprocessing, or tokenization. The front-end then assigns phonetic transcriptions to each word, and divides and marks the text into prosodic units, like phrases, clauses, and sentences. The process of assigning phonetic transcriptions to words is called text-to-phoneme conversion. Phonetic transcriptions and prosody information together make up the symbolic linguistic representation which is carried out by the front-end. The back-end often referred to as the synthesizer converts the symbolic linguistic representation into sound. Although text-to-speech systems have improved over the past few years, some shortcomings still exist. For instance, many text-tospeech systems are designed for only a single language. However, there are many applications that need a system that can provide speech synthesis of words from multiple languages, and in particular, speech synthesis where words from two or more languages are contained in the same sentence. In fact when we look at newspapers we can find many sentences with embedded foreign language expressions or proper names. It would not be appropriate to have these sentences pronounced with a speech engine developed for a single language. The paper is proposed to specifically address the issues of building synthetic voices for the three major languages namely English, Tamil and Telugu.

The techniques employed for synthesizing speech from text may be broadly classified into three categories: 1) Formant-based, 2) Parameter-based and 3) Concatenation-based. The three sub-categories of Concatenationbased are 1) Unit Selection Synthesis, 2) Diphone Synthesis and 3) Domain-Specific Synthesis. We use unit selection synthesis [1] in which, the prerecorded words are split into syllables and stored with the corresponding syllable names and maintained in a database. Based on the input, the syllable units are selected from the database and concatenated to generate the speech.

To make the speech sound more natural Prosody and 
Smoothing is applied at right places. Various techniques available for smoothing include Spectral Smoothing, Optimal Coupling, Waveform Interpolation, LP Techniques and Pitch Synchronous Overlap Add (PSOLA) [2]. We use a technique based on Optimal Coupling algorithm.

The most important qualities of a speech synthesis system are naturalness and Intelligibility. Naturalness describes how closely the output sounds like human speech, while intelligibility is the ease with which the output is understood. The ideal speech synthesizer is both natural and intelligible. Speech synthesis systems usually try to maximize both characteristics. The technologies for generating synthetic speech waveforms are listed below.

1) Concatenative synthesis

a) Unit selection synthesis

b) Diphone synthesis

c) Domain-specific synthesis

2) Formant synthesis

3) Articulatory synthesis

4) HMM-based synthesis

5) Sinewave synthesis

Each technology has its own strengths and weaknesses, and the intended uses of a synthesis system will typically determine which approach is used. In Thirukkural Textto-Speech Synthesis System [3], Concatenative synthesis approach is used where natural speech is concatenated to give the resulting speech output. It involves two phases, namely, the offline phase and the online phase. Offline phase includes pre-processing, segmentation and pitch marking. Online phase includes text analysis and synthesis. Here, syllables have been used as basic units. The size of the database used is high. Attempts are to be made to make the speech sound more natural, add emotions and also to provide good synthesis for alien words.

Formant-based speech synthesizer employing demisyllable concatenation [4], involves identifying and extracting the formants from an actual speech signal (labeled to identify approximate demi-syllable areas) and then using this information to construct demi-syllable segments each represented by a set of filter parameters and a source signal waveform. The basic unit being demi-syllable requires numerous concatenation points and hence the speech lacks from continuous flow.

In Hidden Markov Model (HMM) based speech synthesis system [5] the speech waveform is generated from HMMs themselves, and applies it to English speech synthesis using the general speech synthesis architecture of Festival Framework. Although synthesized speech has a typical quality of "vocoded speech," it has been shown that the mixed excitation model based on Mixed Excitation LPC Vocoder technique (MELP) and post filtering can improve the speech quality significantly.
In Dhvani TTS system [6], they use phoneme concatenation technology with an attempt to cover all Indian languages under a single framework. This system can detect the languages and it dispatches the text to the corresponding phonetic synthesizer. Here, prosody is not given much importance and also the quality of the speech can be improved to some extent. We have proposed a Multilingual Speech Synthesizer to overcome the drawbacks of these systems to the extent as possible.

The paper is organized into 3 sections. In Section 2, we discuss on the proposed system with the description of modules. In Section 3, the results are discussed.

\section{Multilingual TTS System}

The TTS system comprises modules for Preprocessing, Unicode Conversion, Segmentation, Concatenation, Prosody and Smoothing. Figure 1 illustrates the overall architecture of TTS system.

\subsection{Preprocessing}

The first stage of a TTS system is the pre-processing module, called tokenization. It converts the input text into a sequence of words and symbols to be processed by the rest of the system. It identifies and makes decisions on what to do with punctuation marks and other non-alp habetic textual symbols (e.g. parentheses), identifies and expands abbreviations, acronyms, and numbers to fullblown orthographic strings. Each input line is scanned and each recognized construct (word, number, symbol, etc) is converted into an appropriate word or sequence of words as shown in Figure 2.

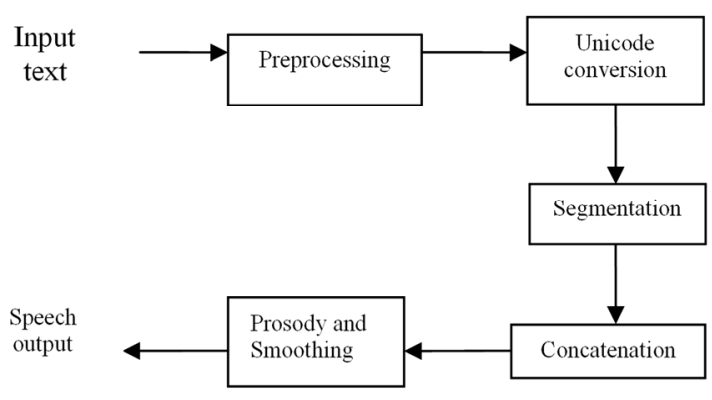

Figure 1. Overall block diagram of the TTS system

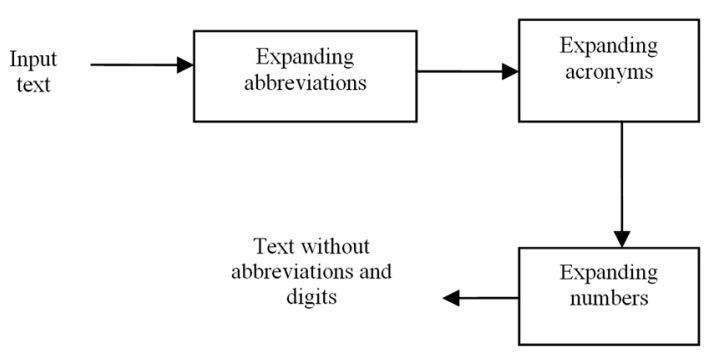

Figure 2. Preprocessing 
Preprocessing includes,

1) The removal of single and double quotes from the start and end of the token.

2) Expansion of abbreviations, acronyms, and numbers.

3) In the case of Tamil and Telugu input, if there is any English word, it is replaced by an equivalent word in the corresponding language.

\subsection{Unicode Conversion}

Unicode is an industry standard allowing computers to consistently represent and manipulate text expressed in most of the world's writing systems. In our work, we have used Unicode conversion to accept the input text in multiple fonts. The logic behind Unicode conversion is to identify the font in which the given text is encoded and applying Unicode encoding to the given font.

For Tamil, since the input may be in TAM, TAB or TSCII, the encoding techniques for TAM, TAB and TSCII are explored and for each and every character in the input, Unicode encoding is applied to convert the given text into unicode. Similarly for Telugu, the encoding technique of shree font is explored and Unicode encoding is applied to the input. In case of multilingual input, the text to be given is already converted to unicode and saved as a text file containing inputs from all the three languages. If the input is going to be in a single language then the Unicode conversion will be carried out internally as shown in Figure 3.

\subsection{Segmentation}

Speech in Indian language is based on basic sound units which are inherently syllable units made from $\mathrm{C}, \mathrm{CV}$, $\mathrm{CCV}$, $\mathrm{VC}$ and $\mathrm{CVC}$ combinations, where $\mathrm{C}$ is a consonant and $\mathrm{V}$ is a vowel. From perceptual results, it is observed that from four different choices of speech units like syllable, diphone, phone and half phone, the syllable unit performs better than all the rest and is a better representation for Indian languages.

In our work, for English, we segment the text into syllables using the basic syllable pattern (C) (C) (C) V (C) (C) (C) (C). Hence, the possible syllable patterns are one among V, CV, CCV, CCV, VC, VCC, VCCC, CVC, VVC, CCVC, CCVCC, CCCVC.

\subsubsection{Rules for Segmentation of English}

1) If a word follows a basic syllable pattern then it is not split into syllables.

2) If a word contains more than one syllable pattern we frame rules based on the pronunciation like,

3) If $\mathrm{CV}$ is followed by $\mathrm{CCV}$ then split the word as $\mathrm{CVC}$ and $\mathrm{CV}$.

4) If $\mathrm{CV}$ is followed by $\mathrm{VC}$ then split the word as $\mathrm{CV}$ and $\mathrm{VC}$.

For Tamil and Telugu, the basic syllable pattern is

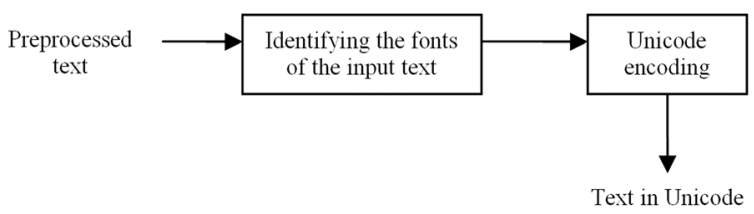

Figure 3. Unicode conversion module



Figure 4. Segmentation of text into syllables

Table 1. Word count for all the three languages

\begin{tabular}{ccc}
\hline Language & No. of Words & Syllable count \\
\hline English & 4200 & 2225 \\
Tamil & 4213 & 1120 \\
Telugu & 4118 & 1950 \\
\hline
\end{tabular}

represented in the form of (C) V (C) (C) and the possible syllable patterns are $\mathrm{V}, \mathrm{CV}, \mathrm{CVC}, \mathrm{CVCC}, \mathrm{VC}, \mathrm{VCC}$ [7]

\subsubsection{Rules for Segmentation of Tamil and Telugu}

1) If characters after $\mathrm{CV}$ pattern are of type $\mathrm{CV}$ then the syllables are split as CV and CV.

2) If the CV pattern is followed by CCV then syllables are split as $\mathrm{CVC}$ and $\mathrm{CV}$.

3) If the $\mathrm{CV}$ pattern is followed by CCCV then syllables are split as CVCC and CV.

4) If the $\mathrm{VC}$ pattern is followed by $\mathrm{V}$ then syllables are split as $\mathrm{V}$ and $\mathrm{CV}$.

5) If the VC pattern is followed by CVC then syllables are split as VC and CVC.

Based on these rules the text is split into syllables and the location of the corresponding sound files for the syllables are written in a text file.

\subsubsection{Collection of Syllables (Speech)}

After segmentation, recording is done at word level and segmented into syllables manually. The recording is done at a frequency of $48 \mathrm{KHz}$ and the channel type is set as Stereo. The syllable count is reduced by finding the syllables which are common for Tamil and Telugu. Eg: Consider the following words:

"Alochinchadam" in Telugu and "alosanai" in Tamil. The syllables for these words are as follows Alochincha dam-a/lo/chin/cha/dam Alosanai -a/lo/sa/nai. In this case, the syllables ' $a$ ' and 'lo' are common for both the lan- 
guages. Hence these syllables are stored only once in the database which can be accessed by both the languages and thereby reduces the size of the database.

\subsubsection{Collection of Input Text}

We collected the input text from different sites

Telugu: www.saakshi.com

Tamil: www.dinamani.com

English: www.hindu.com

Domain: Nuclear deal

The following table gives details about the count of the words and syllables for the three languages.

Syllables common between Tamil and Telugu: 323

\subsection{Concatenation}

In concatenative speech synthesis, the segments of recorded speech are concatenated to produce the desired output [8]. Generally, this technique produces the most natural-sounding synthesized speech since the number of concatenating points is less. There are three main subtypes of concatenative speech synthesis namely Unit selection synthesis and Diphone synthesis. In our work, we use unit selection synthesis in which, the prerecorded words are split into syllables and are stored with the corresponding syllable names and maintained in a database. In case of Tamil and Telugu, the syllable units are named using English letters based on the pronunciation in the corresponding language. For eg., the syllables in the word "வேண்டும்" are named as follows:

$$
\begin{aligned}
& \text { வேண் - veeinn } \\
& \text { டும் - duim }
\end{aligned}
$$

Based on the input, the syllable units are selected from the database and concatenated using MATLAB simulator integrated with java to create complete utterances as shown in Figure 5.

\subsection{Prosody and Smoothing}

\subsubsection{Prosody}

Prosody is the rhythm, stress, and intonation of speech. Prosody may reflect the emotional state of a speaker; whether an utterance is a statement, a question, or a command; whether the speaker is being ironic or sarcastic; emphasis, contrast and focus; and other elements of language which may not be encoded by grammar. Hence, applying the concept of prosody to the TTS system makes the synthesis speech sounds more like human speech. There are several techniques used for prosody $[9,10]$. We make use of the punctuation marks like '!', '?', ';' to give stress. In this method, if the words in the input text are followed by any of these special symbols they are read with intonations. For this method to work, the syllables which are to be read with intonation are maintained as a separate database and these syllable units are

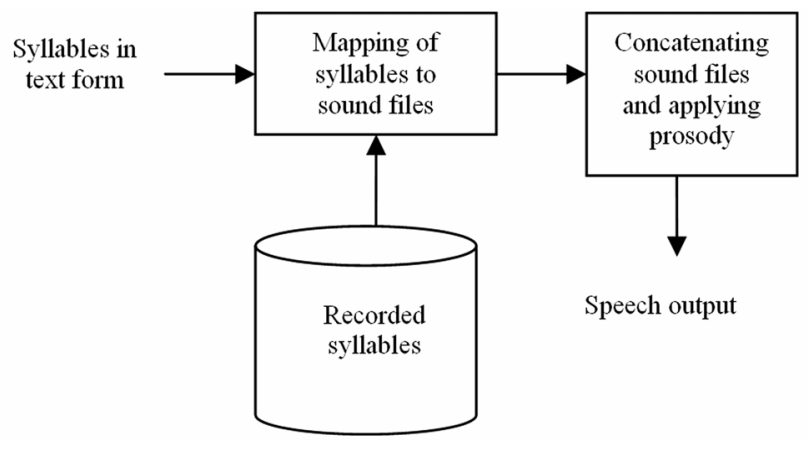

Figure 5. Concatenation using unit selection synthesis

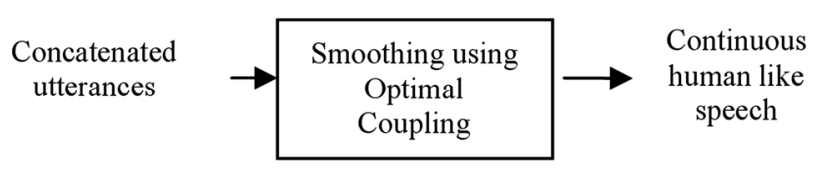

Figure 6. Smoothing

concatenated when intonation is to be given.

\subsubsection{Smoothing}

Smoothing is used to smooth the transition between segments in order to produce continuous output as that produced by human speech as shown in Figure 6.

There are several techniques used for smoothing like Spectral Smoothing, Optimal Coupling, Waveform Interpolation, LP Techniques and Pitch Synchronous Overlap Add (PSOLA). Among all these techniques optimal coupling algorithm is easy to implement at low cost.

It is common in concatenative synthesis that the boundaries of speech segments are fixed, but the optimal coupling technique allows the boundaries to move to provide the best fit with adjacent segments. A measure of mismatch is tested at a number of possible segment boundaries until the closest match is found. While any form of measure may be used, for the sake of improving spectral quality, using a spectral discontinuity measure is appropriate. Measures considered include melfrequency cepstral coefficients (MFCC) [11] and the auditory-neural based measure (ANBM) [12]. It is not necessary to implement optimal coupling to perform spectral smoothing, but it does provide some improvement at a small cost.

We proposed a new technique for Smoothing based on Optimal Coupling algorithm. As shown in Figure 7, initially we set the boundaries to search for the optimal concatenating point. If the given syllable unit is the beginning of the word then the boundary is set from $5 n / 6$ th position to $\mathrm{n}$ where, $\mathrm{n}$ is the length of the syllable unit. Otherwise the boundary is set from the beginning to $\mathrm{n} / 3$ rd position. Then the Hamming windows are formed to process on the selected portions to find the concate- 


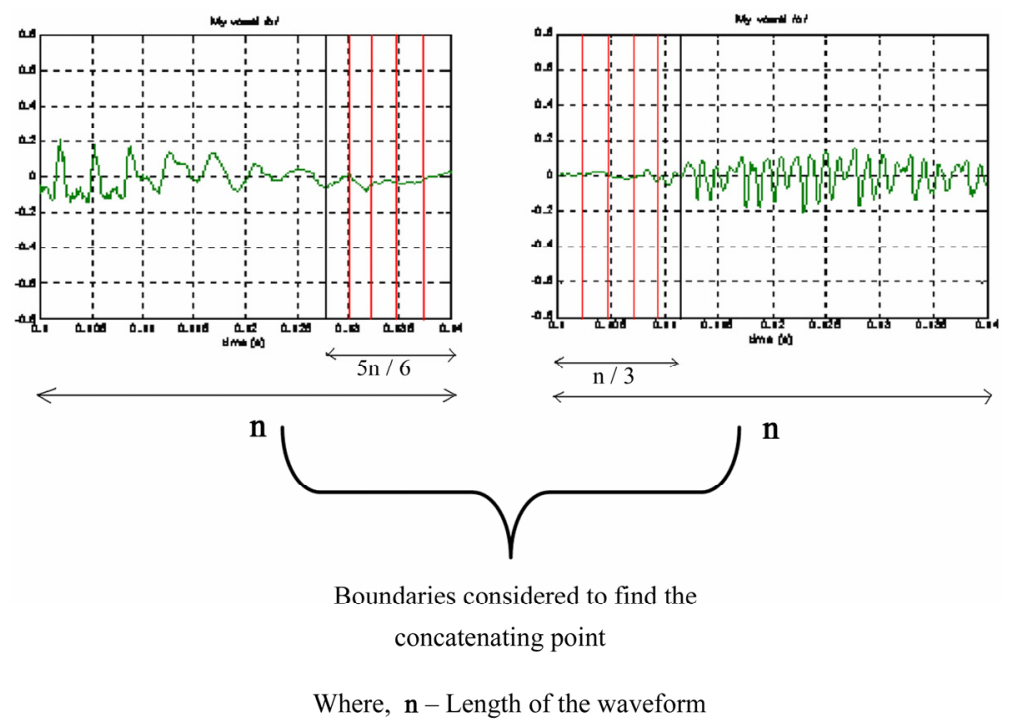

Figure 7. Smoothing using optimal coupling

nating point. The zero-crossing counts are found for all the hamming windows and the point in the area containing the minimum count is chosen as the optimal concatenating point. Finally the syllables are concatenated at the chosen points to generate the smoothened speech output.

\section{Results and Analysis}

The results are analyzed using two parameters namely Mel-Frequency Cepstral Coefficients (MFCCs) and Mean Opinion Score (MOS).

\subsection{Mel-Frequency Cepstral Coefficient (MFCC)}

Mel-frequency cepstral coefficients (MFCCs) are coefficients that collectively make up an MFC. They are derived from a type of cepstral representation of the audio clip. The difference between the cepstrum and the MelFrequency Cepstrum is that in the MFC, the frequency bands are equally spaced on the mel scale, which approximates the human auditory system's response more closely than the linearly-spaced frequency bands used in the normal cepstrum. This frequency warping can allow for better representation of sound, for example, in audio compression. In sound processing, the Mel-Frequency Cepstrum (MFC) is a representation of the short- term power spectrum of a sound, based on a linear cosine transform of a log power spectrum on a nonlinear mel scale of frequency.

MFCCs are commonly derived as follows:

1) Take the Fourier transform of (a windowed excerpt of) a signal.

2) Map the powers of the spectrum obtained above onto the mel scale, using triangular overlapping win- dows.

3) Take the logs of the powers at each of the mel frequencies.

4) Take the discrete cosine transform of the list of mel $\log$ powers, as if it were a signal.

5) The MFCCs are the amplitudes of the resulting spectrum.

MFCCs are commonly used as features in speech recognition systems, such as the systems which can automatically recognize numbers spoken into a telephone.

They are also common in speaker recognition, which is the task of recognizing people from their voices. The MFCC values are calculated for a set of original words say, and the concatenated words. The Euclidean distance between the values of $\mathrm{P}$ and $\mathrm{Q}$ are calculated using the formula,

$$
\begin{aligned}
& \sqrt{\left(p_{1}-q_{1}\right)^{2}+\left(p_{2}-q_{2}\right)^{2}+\cdots+\left(p_{n}-q_{n}\right)^{2}} \\
& =\sqrt{\sum_{i=1}^{n}\left(p_{i}-q_{i}\right)^{2}}
\end{aligned}
$$

The results for English, Tamil and Telugu are tabulated in Table 2,3 and 4 respectively.

The deviation of the concatenated words with the original words in English, Tamil and Telugu based on their MFCC features are plotted in Figures 8,9, and 10 respectively. The average deviation of MFCC value for concatenated English words is 1.398, for Tamil 1.463, and for Telugu 1.492 .Though smoothing technique works well for all the three languages minimum deviation is obtained for English.

\subsection{Mean Opinion Score (MOS)}

The Mean Opinion Score (MOS) provides a numerical 
indication of the perceived quality of received media after compression and/or transmission. The MOS is expressed as a single number in the range 1 to 5 , where 1 is lowest perceived audio quality, and 5 is the highest perceived

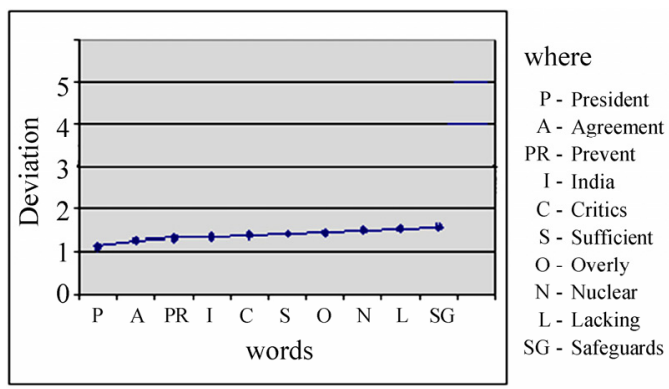

Figure 8. Words (English) vs deviation based on MFCC

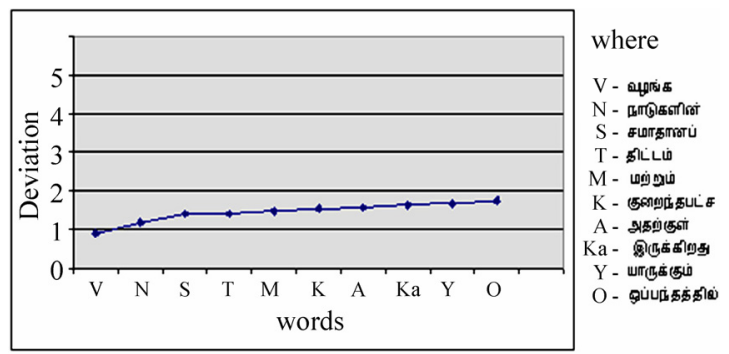

Figure 9. Words (Tamil) vs deviation based on MFCC
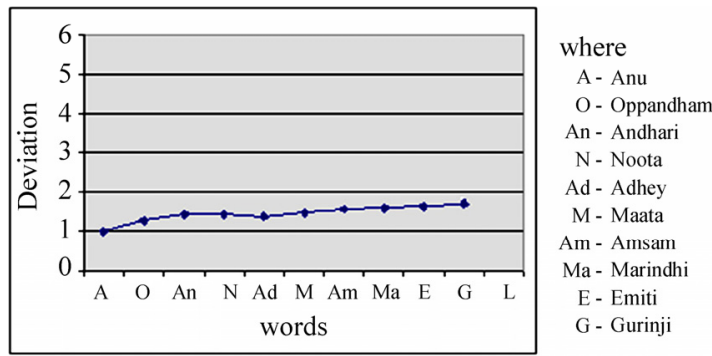

Figure 10. Words (Telugu) vs deviation

Table 2. Result analysis for English using MFCC

\begin{tabular}{cc}
\hline Words & Deviation from the original word \\
\hline President & 1.13 \\
Agreement & 1.26 \\
Prevent & 1.33 \\
India & 1.35 \\
Critics & 1.40 \\
Sufficient & 1.41 \\
Overly & 1.46 \\
Nuclear & 1.51 \\
Lacking & 1.56 \\
Safeguards & 1.57 \\
\hline
\end{tabular}

Table 3. Result analysis for Tamil using MFCC

\begin{tabular}{cc}
\hline Words & $\begin{array}{c}\text { Deriation from the origi- } \\
\text { nal word }\end{array}$ \\
\hline வழங்க & 0.90 \\
நாடுகளின் & 1.20 \\
சமாதாணப் & 1.41 \\
தட்டம் & 1.43 \\
மற்றும் & 1.50 \\
குறைந்தபட்ச & 1.56 \\
அதற்கள் & 1.57 \\
இருக்கிறது & 1.65 \\
யாருக்கும் & 1.68 \\
ஒய்ந்தத்தில் & 1.73 \\
\hline
\end{tabular}

Table 4. Result analysis for Telugu using MFCC

\begin{tabular}{|c|c|}
\hline Words & Deviation from the original \\
\hline అణు & 1.0 \\
\hline ఒపపరందంం & 1.30 \\
\hline అందరి & 1.44 \\
\hline șట & 1.46 \\
\hline అదే & 1.40 \\
\hline మాట & 1.50 \\
\hline అంశం & 1.58 \\
\hline మారిది & 1.60 \\
\hline ఎమిటి & 1.65 \\
\hline గుంంచి & 1.70 \\
\hline
\end{tabular}

Table 5. Rating of MOS

\begin{tabular}{ccc}
\hline & \multicolumn{2}{c}{ Mean opinion score (MOS) } \\
\hline MOS & Quality & Impairment \\
\hline 5 & Excellent & Imperceptible \\
4 & Good & Perceptible but not annoying \\
3 & Fair & Slightly annoying \\
2 & Poor & Annoying \\
1 & Bad & Very annoying \\
\hline
\end{tabular}

Table 6. Results of mean opinion score

\begin{tabular}{cccc}
\hline \multirow{2}{*}{ LISTENERS } & \multicolumn{3}{c}{ MOS } \\
\cline { 2 - 4 } & English & Tamil & Telugu \\
\hline Male (28) & 3.9 & 3.7 & 3.5 \\
Female (40) & 4.0 & 3.9 & 3.6 \\
\hline
\end{tabular}

audio quality measurement. The MOS is generated by averaging the results of a set of standard, subjective tests where a number of listeners rate the heard audio quality of test sentences read aloud by both male and female speakers over the communications medium being tested. A listener is required to give each sentence a rating using the rating scheme as shown in Table 5 and MOS is the arithmetic mean of all the individual scores, and can range from 1 (worst) to 5 (best). We took objective measurements from listener tests. Sixty expert listeners 
were asked to indicate their preferences in terms of naturalness and intelligibility for different words and phrases. Table 6 shows the preliminary results of Mean Opinion Score (MOS).

The listeners ranked the smoothened speech produced after concatenation as compared with natural speech. The results taken using these parameters signify that the speech is generated with minimal distortion.

\section{Conclusions}

The system has been successfully developed for the three languages Indian English, Tamil and Telugu. Syllable based concatenation was used which reduced the concatenation points and hence minimal distortion. Optimal Coupling technique has been used for Smoothing which resulted in natural sounding speech. The synthesizer was tested for its quality through expert listeners. In the proposed work we process only domain restricted text. In future the Synthesizer has to be enhanced to process unrestricted text and the Smoothing technique needs to be explored further to find the optimal concatenating point. Also more Indian languages have to be included.

\section{References}

[1] S. P. Kishore, R. Kumar, and R. Sangal "A data-driven synthesis approach for Indian languages using syllable as basic unit," in Intl. Conf. on Natural Language Processing (ICON), pp. 311-316, 2002.

[2] D. T. Chappell and J. H. L. Hansen "Spectral smoothing for speech segment concatenation," Speech Communication, Vol. 36, No. 3-4, 2002.

[3] G. L. Jayavardhana Rama, A. G.Ramakrishnan, M. Vijay Venkatesh and R. Murali Shankar, "Thirukkural-A textto-speech synthesis system," Paper Presented in the the
Tamil Internet 2001 Conference and Exhibition (TI2001), 2001.

[4] S. Schötz, "Data-driven formant synthesis of speaker age," In G. Ambrazaitis and S. Schötz (eds.). Lund Working Papers 52, Proceedings of Fonetik, Lund, pp. 105-108. 2006

[5] K. Tokuda, H. Zen, and A. W. Black, "An hmm-based speech synthesis system applied to English," paper Presented in the Proc. of IEEE Speech Synthesis Workshop, 2002 .

[6] http://dhvani.sourgeforge.net, (Dhvani-TTS System for Indian Languages), 2001.

[7] S. Saraswathi and T. V. Geetha, "Language models for Tamil Speech Recognition," Publication in IETE Special Issue on Spoken Language Processing, Vol. 24, No. 5, pp. 375-383, 2007.

[8] Céu Viana, "Concatenative speech synthesis for European Portuguese," Paper Presented in the third ESCA/COSCOSDA International Workshop on Speech Synthesis, Australia, 1998.

[9] N. Sridhar Krishna and H. A. Murthy, "Duration modeling of Indian languages Hindi and Telugu," Paper Presented in the proceedings of 5th ISCA Speech Synthesis Workshop, 2004.

[10] N. Sridhar Krishna and H. A. Murthy, "A new prosodic phrasing model for Indian language Telugu", Paper Presented in the Proceedings of Interspeech-2004, ICSLP 8th International Conference on Spoken Language Processing, pp. 793-796, 2004.

[11] S. Imai, "Cepstral analysis synthesis on the mel frequency Scale," Paper Presented in Proceedings of ICASSP, Vol. 8, pp. 93-96, 1983.

[12] J. H. L. Hansen and D. T. Chappell, "An auditory based distortion measure with application to concatenative speech synthesis," IEEE Transactions on Speech and Audio Processing, Vol. 6, No. 5, pp. 489-495, ge and Data Engineering, 1999, Vol. 11, No. 1, pp. 133-142, 1998. 